Vietnam Journal of Mechanics, NCST of Vietnam Vol. 24, 2002, No2 (101-114)

\title{
SUCCESSIVE ALGORITHM FOR CONSTRUCTION OF EQUATION OF MOTION OF CONSTRAINED MECHANICAL SYSTEMS
}

\author{
DINH VAN PHONG \\ Department of Applied Mechanics, Hanoi University of Technology
}

\begin{abstract}
The article devotes to the construction of equations of motion of constrained mechanical systems. The constraint conditions are successively appended to the already defined systems. Therefore the algorithm is very flexible and allows studying the separate constraints more in detail. For illustration of consequent steps of the algorithm one simple example is shown.
\end{abstract}

\section{Introduction}

Nowadays in technical application the constraints of mechanical systems are becoming increasingly important. They appear in the complex systems such as machines, robots and other multibody systems where the constraints can introduce the interaction between mechanical problems and problems of other characters: control, electric and hydraulic law etc. Even for purely mechanical systems using constraints occurs when the redundant generalized coordinates are chosen.

The equation of motion of constrained mechanical systems is mostly derived by using Lagrange multipliers. In combination with various methods, e.g. NewtonEuler equations or Kane's equations for multibody systems etc., the full form or reduced forms of equation of motion are available. In the reduced form Lagrange multipliers are excluded from the equation. With the progress of computer technology recursive methods are also developed, see e.g. [7], [10], [11], [13] etc.

In this article we will develop one new method for constructing the equation of motion of constrained mechanical systems. The idea of the method is based on the principle of compatibility, see e.g. [4], [5]. The equation of motion for the free system, i.e. the system without constraints, is assumed to have been constructed before. Then the constraints can be added successively to the existing system.

Since there exists a lot of methods for constructing the equation of motion of unconstrained systems, this successive algorithm enables us to study the influence of constraints in the more flexible way and more in detail. The algorithm is suggested for computer processing. However, in the illustrative example we can see that manual handling is possible, too. 


\section{Theoretical background}

Consider the system with $n$ generalized coordinates, which are the components of a $n$-dimensional vector $\mathbf{q}$. The equation of motion is written in the form:

$$
\mathbf{A} \ddot{\mathbf{q}}=\mathbf{h}+\mathbf{r}
$$

where $\mathbf{A}$ is the system mass matrix of dimension $(n \times n), \ddot{\mathbf{q}}$ is the $n$-dimensional vector of generalized accelerations, $\mathrm{h}$ is the $n$-dimensional vector of all forces acting on the system that also includes Coriolis and centrifugal forces but not constraint forces, $\mathbf{r}$ is the $n$-dimensional vector of constraint forces due to constraints applied to the system.

Assume that the constraints are written in the matrix form:

$$
\mathrm{G} \ddot{\mathrm{q}}+\mathrm{g}_{o}=0
$$

where $\mathbf{G}$ is the $(s \times n)$-dimensional coefficient matrix, $\mathbf{g}_{o}$ is a $s$-dimensional vector and $s$ is the number of constraints. $\mathbf{G}$ and $\mathbf{g}_{o}$ are, in general, functions of the vector of generalized coordinates $\mathbf{q}$ and the vector of generalized velocities $\dot{\mathbf{q}}$.

We divide $s$ constraint equations into two groups, with $s_{1}$ respectively $s_{2}$ constraints in each group:

$$
\begin{aligned}
& \mathrm{G}_{1} \ddot{\mathbf{q}}+\mathrm{g}_{1}=\mathbf{0} \\
& \mathrm{G}_{2} \ddot{\mathbf{q}}+\mathrm{g}_{2}=\mathbf{0}
\end{aligned}
$$

where $\mathbf{G}_{1}, \mathbf{G}_{2}$ are the matrices of the dimension $\left(s_{1} \times n\right)$ or $\left(s_{2} \times n\right)$ respectively, $\mathbf{g}_{1}$ and $\mathbf{g}_{2}$ are vectors of dimension $s_{1}$ or $s_{2}$ respectively. As shown in [2] each constraint has a corresponding term in the total reaction force $\mathbf{r}$, hence the corresponding reaction forces of the group 1 respectively 2 can be denoted as $\mathbf{r}_{1}$ and $\mathbf{r}_{2}$ respectively. Obviously the following holds:

$$
\mathbf{r}=\mathbf{r}_{1}+\mathbf{r}_{2}
$$

Therefore the equation (2.1) is written in the form:

$$
\mathbf{A} \ddot{\mathbf{q}}=\mathbf{h}+\mathbf{r}_{1}+\mathbf{r}_{2} .
$$

From (2.6) we have:

$$
\ddot{\mathbf{q}}=\mathbf{A}^{-1} \mathbf{h}+\mathbf{A}^{-1} \mathbf{r}_{1}+\mathbf{A}^{-1} \mathbf{r}_{2}
$$

Putting $\ddot{\mathbf{q}}$ from (2.7) into the equations (2.3) and (2.4) yields:

$$
\begin{aligned}
& \mathbf{G}_{1} \mathbf{A}^{-1} \mathbf{r}_{1}+\mathbf{G}_{1} \mathbf{A}^{-1} \mathbf{r}_{2}+\mathbf{G}_{1} \mathbf{A}^{-1} \mathbf{h}+\mathbf{g}_{1}=\mathbf{0} \\
& \mathbf{G}_{2} \mathbf{A}^{-1} \mathbf{r}_{1}+\mathbf{G}_{2} \mathbf{A}^{-1} \mathbf{r}_{2}+\mathbf{G}_{1} \mathbf{A}^{-1} \mathbf{h}+\mathbf{g}_{2}=\mathbf{0} .
\end{aligned}
$$


Now we will find out the influence of constraint reaction forces $\mathbf{r}_{1}$ and $\mathbf{r}_{2}$ to each other. Assume that $\mathbf{r}_{2}$ is known and the task is calculating $\mathbf{r}_{1}$. From (2.8) one obtains:

$$
\mathbf{G}_{1} \mathbf{A}^{-1} \mathbf{r}_{1}=-\mathbf{G}_{1} \mathbf{A}^{-1} \mathbf{r}_{2}-\mathbf{G}_{1} \mathbf{A}^{-1} \mathbf{h}-\mathbf{g}_{1} .
$$

With assumption of ideal constraints we have $n-s_{1}$ equations:

$$
\mathbf{D}_{1}^{T} \mathbf{r}_{1}=0
$$

where $\mathbf{D}_{1}$ is the $\left(n \times\left(n-s_{1}\right)\right)$-dimensional matrix, see [3].

The system of equation (2.10) and (2.11) gives $n$ algebraic equations for $n$ unknowns: the components of the vector $r_{1}$. Under the assumption of well-chosen constraints, it means:

$$
\operatorname{rank}\left[\begin{array}{c}
\mathbf{G}_{1} \mathbf{A}^{-1} \\
\mathbf{D}_{1}^{T}
\end{array}\right]=n,
$$

the system of (2.10) and (2.11) yields the reaction forces $\mathbf{r}_{1}$. It is necessary to emphasize that the first term of the right of (2.10) requires the values of reaction force $\mathbf{r}_{2}$.

We will analyze this result more in detail. Consider the situation when we at first apply to the system the first group of $s_{1}$ constraints. The corresponding reaction force is $\mathbf{r}_{1}^{(0)}$. In the second stage the second group of $s_{2}$ constraint will be applied to the system. Obviously the reaction forces of the first group will be changed in this new situation. The change of reaction force of the first group is denoted by $\Delta \mathbf{r}_{1}$. The total reaction force of the first group is $\mathbf{r}_{1}$ as we have defined before. We can write:

$$
\mathbf{r}_{1}=\mathbf{r}_{1}^{(0)}+\Delta \mathbf{r}_{1}
$$

It is interesting that both terms $\mathbf{r}_{1}^{(0)}$ and $\Delta \mathbf{r}_{1}$ could be calculated from the system of equation (2.10) and (2.11). Really, $\mathbf{r}_{1}^{(0)}$ depends only on the constraints of the first group and the term $\Delta \mathbf{r}_{1}$ depends on the reaction force $\mathbf{r}_{2}$. Consequently, the system of equations (2.10) and (2.11) is split into two subsystems:

$$
\begin{aligned}
\mathbf{G}_{1} \mathbf{A}^{-1} \mathbf{r}_{1}^{(0)} & =-\mathrm{G}_{1} \mathbf{A}^{-1} \mathbf{h}-\mathrm{g}_{1} \\
\mathbf{D}_{1}^{T} \mathbf{r}_{1}^{(0)} & =\mathbf{0}
\end{aligned}
$$

and

$$
\begin{aligned}
\mathbf{G}_{1} \mathbf{A}^{-1} \Delta \mathbf{r}_{1} & =-\mathbf{G}_{1} \mathbf{A}^{-1} \mathbf{r}_{2} \\
\mathbf{D}_{1}^{T} \Delta \mathbf{r}_{1} & =0
\end{aligned}
$$


Theoretically, $\Delta \mathbf{r}_{1}$ could be calculated from (2.15) and (2.16), if $\mathbf{r}_{2}$ is known. The following lemma gives the formal relation of $\Delta \mathbf{r}_{1}$ and $\mathbf{r}_{2}$.

Lemma. From (2.15) and (2.16), $\Delta \mathbf{r}_{1}$ can be determined as follows:

$$
\Delta \mathbf{r}_{1}=\mathrm{Cr}_{2}
$$

where $\mathbf{C}$ is a $(n \times n)$-dimensional matrix. The elements of $\mathbf{C}$ could be evaluated as:

$$
c_{i j}=\sum_{\alpha=1}^{s_{1}} f_{\alpha j} \frac{\Delta_{\alpha i}}{\Delta}, \quad i, j=1, \ldots, n,
$$

where $\Delta$ is the determinant of the matrix $\mathbf{B}$, defined as

$$
\mathbf{B}=\left[\begin{array}{c}
\mathbf{F} \\
\mathbf{D}_{1}^{T}
\end{array}\right], \text { with } \quad \mathbf{F}=\mathbf{G}_{1} \mathbf{A}^{-1}
$$

$f_{\alpha j}$ are elements of the matrix $\mathbf{F}$

$\Delta_{\alpha i}$ is algebraic complement of the system (2.15) and (2.16).

Proof. Consider the linear algebraic system of equations (2.15) and (2.16) which is written in the form:

$$
\mathbf{B} \mathbf{x}=-\mathbf{B}^{\prime} \mathbf{y}
$$

where for the sake of simplicity we denote

$$
\begin{aligned}
& \mathbf{x}=\Delta \mathbf{r}_{1}, \\
& \mathbf{y}=\mathbf{r}_{2}
\end{aligned}
$$

and the matrix $\mathbf{B}^{\prime}$ has the form

$$
\mathbf{B}^{\prime}=\left[\begin{array}{c}
-\mathbf{F} \\
0
\end{array}\right]
$$

Applying the Crammel's law to the system yields:

$$
x_{i}=-\frac{\sum_{\alpha=1}^{n}\left(\sum_{j=1}^{n} b_{\alpha j}^{\prime} y_{j}\right) \Delta_{\alpha i}}{\Delta} .
$$

Since the right side of $(2.20)$ has the special form (2.21) with last rows including only zeros, one obtains:

$$
x_{i}=-\frac{\sum_{\alpha=1}^{s_{1}}\left(\sum_{j=1}^{n} b_{\alpha j} y_{j}\right) \Delta_{\alpha i}}{\Delta} \cdot \text { Consequently, } \quad x_{i}=-\sum_{j=1}^{n}\left(\sum_{\alpha=1}^{s_{1}} \frac{b_{\alpha j} \Delta_{\alpha i}}{\Delta}\right) y_{j} .
$$


Denoting the term in the bracket of the last equations as $c_{i j}$ completes the proof of the lemma.

Above theoretical consideration suggests that we can study at first the situation when only one group of constraints affects the system. The equation of motion is derived for this configuration. In the second stage, the second group of constraints is applied to the system. Formally, the equation of motion, which has the form (2.1), has unchanged structure. Only the ratio between the terms of $\mathbf{r}_{1}$ and $\mathbf{r}_{2}$ appears as we have shown above. Roughly speaking, by adding more constraints the reaction force $\mathbf{r}$ should be incremented by two terms:

- the reaction force $\mathbf{r}_{2}$ of the second group of constraints;

- the increment of reaction force of the first group that is denoted above as $\Delta \mathbf{r}_{1}$. Obviously the constraint equations and the equations derived from the condition of ideality of the constraints will change their structures.

The change of constraint equations is observed simply. Since $s_{2}$ new constraints are added to the system we have more $s_{2}$ equations. The matrix $\mathrm{G}$, now, has $s_{1}+s_{2}$ rows, contrary to $s_{1}$ rows in the situation when only $s_{1}$ constraints affect the system.

Now consider the change of equations, derived from the condition of ideality of constraints. In order to derive the successive method we can assume that in the second group there is only one new constraint. For the sake of simplicity we denote this equation of constraint by the form:

$$
\mathbf{g} \ddot{\mathbf{q}}+\mathrm{g}_{0}=0 .
$$

Obviously $\mathbf{g}$ is a $n$-dimensional row vector.

In the first stage when the system has only $s_{1}$ constraints, the criterion of ideality yields:

$$
\mathrm{G}_{1} \mathrm{D}_{1}=\mathbf{0}
$$

where $\mathbf{G}_{1}$ is of $\left(s_{1} \times n\right)$ dimension and $\mathbf{D}_{1}$ is of $(n \times m)$ dimension where $m=n-s_{1}$, see [2].

From the previous discussion it is clear that when the new constraint is appended to the system we will have one more constraint equation, i.e. (2.22), and one less equation in the condition of ideality. It means that the matrix $\mathbf{G}$ has one more row and matrix $\mathbf{D}$ has one less row. In this case the relation, similar to (2.23), holds:

$$
\mathrm{GD}=\mathbf{0},
$$

but the matrix $\mathbf{G}$ is of $\left(s_{1}+1\right) \times n$ dimension and the matrix $\mathbf{D}$ is of $n \times(m-1)$ dimension. Clearly, (2.24) could be written in the following form:

$$
\left[\begin{array}{c}
\mathrm{G}_{1} \\
\mathrm{~g}
\end{array}\right] \mathrm{D}=0 .
$$


Since the matrices $\mathrm{D}$ and/or $\mathrm{D}_{1}$ are defined as the set of vectors orthogonal to the rows of matrices $\mathbf{G}$ and/or $\mathbf{G}_{1}$, from (2.23) and (2.24) we can write:

$$
\mathrm{D}=\mathrm{D}_{1} \mathrm{H}
$$

where $\mathbf{H}$ is a $m \times(m-1)$-dimensional matrix. If we can construct the matrix $\mathbf{H}$ by some way, the matrix $\mathbf{D}$ is constructed also easily from (2.26). That is important for us, since $\mathbf{D}_{1}$ is defined in the situation without constraint (2.22) and $\mathbf{D}$ is defined with this constraint.

Replacement D from (2.26) into (2.25) yields:

$$
\left[\begin{array}{c}
G_{1} \\
g
\end{array}\right] D_{1} \mathbf{H}=\mathbf{0}
$$

or

$$
\left[\begin{array}{c}
G_{1} D_{1} \\
\mathrm{~g} \mathrm{D}_{1}
\end{array}\right] \mathbf{H}=\mathbf{0},
$$

and from (2.23) it yields:

$$
\left[\begin{array}{c}
0 \\
\mathrm{~g} \mathrm{D}_{1}
\end{array}\right] \mathrm{H}=\mathbf{0}
$$

This equation gives us the instruction for constructing the matrix $\mathbf{H}$ : the $(m-1)$ columns of the matrix $H_{m \times(m-1)}$ can be defined as a set of vectors orthogonal to the row vector $\mathbf{g} \mathbf{D}_{1}$. As known the Gram-Schmidt orthogonalization could be applied easily for this purpose.

This conclusion means that from the matrix $\mathbf{D}_{1}$, already defined in the previous stage and the constraint vector $\mathbf{g}$ in the consequent stage, we can define matrix $\mathbf{H}$ from (2.27) and consequently, from (2.26) the matrix D. Hence, the equation for determining reaction force $\mathbf{r}$ :

$$
\mathbf{D}^{T} \mathbf{r}=0
$$

is obtained. And the total set of equation of motion consisting of (2.1), (2.2) and (2.28) is available.

The described procedure could be repeated for other constraint. So we can conclude that equation of motion can be constructed from the "free" system when no constraint affects the mechanical system. Then the constraints are added to the system successively, one after one. Since the equation of motion for the free system in general is not so complicated, the main task is the consecutive construction of the matrix $\mathbf{D}$ and/or $\mathbf{H}$ in each step. This construction could be done by computer for the large system.

One another advantage of described technique is in the case when the application is subjected to the additional constraint. It is not necessary to rebuild all parts of 
the equation of motion, but only the matrices $\mathbf{H}$ and $\mathbf{D}$. It means that only one step of the algorithm is done. But the system of equation of motion of the original system, i.e. without the additional constraint, can be constructed by any other techniques. This combination makes the handling more flexible.

\section{Algorithm}

Now we will show the algorithm for constructing the equations of motion by consecutive adding constraints.

Consider the system with $n$ generalized coordinates and $s$ constraints. In the following algorithm these constraints will be added successively to the system under consideration.

\section{Algorithm 1}

1. Construction of equation of motion for free system in the form:

$$
\mathbf{A} \ddot{\mathbf{q}}=\mathbf{h}+\mathbf{r}
$$

2. Setting:

$$
\begin{aligned}
& i=1, \\
& m=n, \\
& \mathbf{D}_{n \times n}=\mathbf{I}_{n \times n} \text { (identity matrix). }
\end{aligned}
$$

3. Adding the constraint:

$$
g_{i}=0
$$

to the system of equation of motion.

4. Calculating the row Jacobian vector $\mathbf{J}_{1 \times n}$ of the constraint $g_{i}$.

5. Calculating the row vector:

$$
\beta=\mathrm{J} \mathrm{D} \text {. }
$$

6. Calculating the matrix $\mathbf{H}_{m \times(m-1)}$ by choosing $(m-1)$ column vectors orthogonal to the row vector $\beta$. The dimension of this vector is $m$ and Gram-Schmidt orthogonalization could be used.

7. Putting:

$$
\mathbf{D}=\mathbf{D} \mathbf{H}
$$

8. Setting:

$$
\begin{aligned}
& i=i+1 \text {, } \\
& m=m-1 \text {, } \\
& \text { if } i \leq s \text { back to the step } 3 \text {, } \\
& \text { otherwise to the step } 9 \text {. }
\end{aligned}
$$

9. Adding the equation:

$$
\mathbf{D}^{T} \mathbf{r}=\mathbf{0} \text {. }
$$

to the system of equations of motion. 
Recall that after running algorithm we get the equation of motion in the form:

$$
\begin{aligned}
& A \ddot{q}=h+r \\
& g(q, \dot{q}, t)=0 \\
& D^{T} \mathbf{r}=0 .
\end{aligned}
$$

In the above algorithm if the reduced form of equations of motion is required, additional multiplying matrices $\mathbf{A}$ and $\mathbf{h}$ by the matrix $\mathbf{H}^{T}$ will yield the required equation.

Really, the reduced form of equation of motion that does not include reaction forces is, see [8]:

$$
\mathbf{D}^{T} \mathbf{A} \ddot{\mathbf{q}}=\mathbf{D}^{T} \mathbf{h} .
$$

In the next consecutive step when one new constraint is appended, the matrix $\mathbf{D}$ is changed by multiplying with the matrix $\mathbf{H}$ (step 7), so the equation (3.2) has the following form:

$$
\mathbf{H}^{T} \mathbf{D}^{T} \mathbf{A} \ddot{\mathbf{q}}=\mathbf{H}^{T} \mathbf{D}^{T} \mathbf{h} .
$$

We can denote:

$$
\begin{aligned}
\mathbf{C} & =\mathbf{D}^{T} \mathbf{A} \\
\mathbf{f} & =\mathbf{D}^{T} \mathbf{h}
\end{aligned}
$$

and modify the above algorithm in order to get the algorithm for constructing the reduced form of equations of motion. It is clear from (3.3) that the coefficient matrix C corresponding to the acceleration $\ddot{\mathrm{q}}$ will be changed by consecutive multiplying with $\mathbf{H}^{T}$ at each step.

\section{Algorithm 2}

1. Construction of matrices $\mathbf{A}, \mathbf{h}$ for free system.

2. Setting:

$$
\begin{aligned}
& i=1, \\
& \mathbf{D}=\mathbf{I}, \\
& \mathbf{C}=\mathbf{A}, \\
& \mathbf{f}=\mathbf{h} .
\end{aligned}
$$

3. Adding the constraint

$$
g_{i}=0
$$

to the system of equation of motion.

4. Calculating the Jacobian row matrix:

$$
\mathbf{J}_{1 \times n}=\frac{\partial g_{i}}{\partial \mathbf{q}} \text {. }
$$

5. Calculating the row matrix: 


$$
\beta=\mathrm{J} \text { D. }
$$

6. Deriving the matrix $\mathbf{H}_{m \times(m-1)}$ by Gram-Schmidt orthogonalization. The columns of $\mathbf{H}$ are orthogonal to $\boldsymbol{\beta}$.

7. Setting:

$$
\begin{aligned}
& \mathbf{D}=\mathbf{D} \mathbf{H} \\
& \mathbf{C}=\mathbf{H}^{T} \mathbf{C} \\
& \mathbf{f}=\mathbf{H}^{T} \mathbf{f} .
\end{aligned}
$$

8. Setting

$$
\begin{aligned}
& i=i+1 \\
& m=m-1 \\
& \text { If } \quad i \leq s \text { back to the step } 3 \\
& \text { otherwise stop. }
\end{aligned}
$$
form:

After running this algorithm we get the system of equations of motion in the

$$
\begin{aligned}
& \mathrm{C} \ddot{\mathbf{q}}=\mathrm{f} \\
& \mathbf{g}(\mathbf{q}, \dot{\mathbf{q}}, \mathbf{t})=0 .
\end{aligned}
$$

It is possible to write both algorithms 1 and 2 in one common algorithm and obtain the matrices $\mathbf{D}, \mathbf{C}, \mathbf{h}$ and $\mathbf{f}$ as the output. The equations of motion are constructed in the form (3.1) or (3.4), depending on the requirement defined at the input. Then the numerical integration could be provided.

For the large systems the algorithm could be done on the computer. However, in the following example we will see that the manual handling is also possible.

\section{Illustrative example}

Consider a planar case of rolling disc without slipping on the rough surface. The radius of the disc is $r$. The mass and the inertia of the disc is $m$ respectively $J$. Three generalized coordinates are chosen to described the motion of the system: $x$, $y$ and $\varphi$, as shown in the figure 1 .

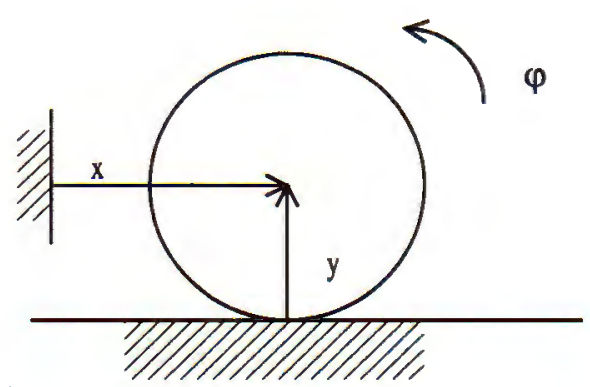

Fig. 1 
At the final stage we will have two constraint conditions $(s=2)$ for this system as follows:

$$
\begin{aligned}
& y-r=0, \\
& x+r \varphi=0 .
\end{aligned}
$$

In order to demonstrate how above algorithms work we will consider, firstly, the free system, when both constraints are released. The system has, hence, 3 degrees of freedom. Each of constraint (3.5) and (3.6) when being appended to the system will reduce one degree of freedom. Now we will apply the derived algorithm to construct the system of equations of motion.

At first for free system without constraints, the equation of motion with 3 generalized coordinates is derived very easily. From the expression of the kinetic energy $T$ and the vector of generalized force $\mathbf{Q}$, we get:

$$
\begin{aligned}
m \ddot{x} & =0 \\
m \ddot{y} & =-m g \\
J \ddot{\varphi} & =0
\end{aligned}
$$

or in the sense of the principle of compatibility

$$
\begin{aligned}
& m \ddot{x}=r_{x} \\
& m \ddot{y}=-m g+r_{y} \\
& J \ddot{\varphi}=r_{\varphi} \\
& r_{x}=0 \\
& r_{y}=0 \\
& r_{\varphi}=0 .
\end{aligned}
$$

Therefore one gets:

$$
\mathbf{A}=\left[\begin{array}{ccc}
m & 0 & 0 \\
0 & m & 0 \\
0 & 0 & J
\end{array}\right], \quad \mathbf{h}=\left[\begin{array}{c}
0 \\
-m g \\
0
\end{array}\right] .
$$

Clearly, at this stage $n=3, s=0, m=3$.

Consider the next stage when the disc is constrained by the constraint (3.5), we have:

$$
\mathbf{D}=\left[\begin{array}{lll}
1 & 0 & 0 \\
0 & 1 & 0 \\
0 & 0 & 1
\end{array}\right]
$$

and from (3.5)

$$
g_{1}=y-r
$$


therefore

$$
\mathbf{J}=\left[\begin{array}{lll}
0 & 1 & 0
\end{array}\right]
$$

That follows:

$$
\beta=\left[\begin{array}{lll}
0 & 1 & 0
\end{array}\right]
$$

The process of Gram-Schmidt orthogonalization yields:

$$
\mathbf{H}_{3 \times 2}=\left[\begin{array}{ll}
1 & 0 \\
0 & 0 \\
0 & 1
\end{array}\right]
$$

Then

$$
\mathbf{D}_{3 \times 2}=\left[\begin{array}{ll}
1 & 0 \\
0 & 0 \\
0 & 1
\end{array}\right], \quad \mathbf{C}=\left[\begin{array}{ccc}
m & 0 & 0 \\
0 & 0 & J
\end{array}\right], \quad \mathbf{f}=\left[\begin{array}{l}
0 \\
0
\end{array}\right]
$$

The equation of motion at this stage when only (3.5) affects the system is in the reduced form:

$$
\begin{aligned}
m \ddot{x} & =0 \\
J \ddot{\varphi} & =0 \\
y-r & =0 .
\end{aligned}
$$

The full form is as follows:

$$
\begin{aligned}
& m \ddot{x}=r_{x} \\
& m \ddot{y}=-m g+r_{y} \\
& J \ddot{\varphi}=r_{\varphi} \\
& y-r=0 \\
& r_{x}=0 \\
& r_{\varphi}=0,
\end{aligned}
$$

where $r_{x}, r_{y}, r_{\varphi}$ are the reaction forces of constraints (3.5). Note at this stage $n=3$, $s=1, m=2$.

In the next stage when (3.6) also affects the system, one gets

$$
\mathbf{J}=\left[\begin{array}{lll}
1 & 0 & r
\end{array}\right]
$$

therefore

$$
\beta=\left[\begin{array}{lll}
1 & 0 & r
\end{array}\right]\left[\begin{array}{ll}
1 & 0 \\
0 & 0 \\
0 & 1
\end{array}\right]=\left[\begin{array}{ll}
1 & r
\end{array}\right]
$$


and Gram-Schmidt orthogonalization yields

$$
\mathbf{H}_{2 \times 1}=\left[\begin{array}{c}
r \\
-1
\end{array}\right]
$$

Then

$$
\mathbf{D}=\left[\begin{array}{c}
r \\
0 \\
-1
\end{array}\right], \quad \mathbf{C}=\left[\begin{array}{lll}
r m & 0 & -J
\end{array}\right], \quad \mathbf{f}=[0]
$$

The system of equations of motion in the full form, with $n=3, s=2, m=1$, is as follows:

$$
\begin{aligned}
& m \ddot{x}=r_{x} \\
& m \ddot{y}=-m g+r_{y} \\
& J \ddot{\varphi}=r_{\varphi} \\
& y-r=0 \\
& x+r \varphi=0 \\
& r_{x} r-r_{\varphi}=0 .
\end{aligned}
$$

Recall that in this system of equation $r_{x}, r_{y}$ and $r_{\varphi}$ are reaction forces, corresponding to both constraints (3.5) and (3.6). They are evaluated in the numerical integration process.

The system of equation of motion can be also written in the following reduced form:

$$
\begin{aligned}
& r m \ddot{x}-J \ddot{\varphi}=0 \\
& y-r=0 \\
& x+r \varphi=0 .
\end{aligned}
$$

In this example we can see the flexibility of our approach. The system of equations of motion is systematically derived for 3 cases:

- free system when no constraint affects the system;

- system with one constraint;

- system with both constraints.

In the constrained systems the equations of motion could be written in the full form including all reaction forces or in the reduced form where the reaction forces are removed. Obviously, with the reduced form the reaction forces can be evaluated later, see e.g. [8]

Recall that the total number of equations of motion is unchanged. In our case with $n=3$, the number of equations in the full form is $2 n=6$, and in the reduced form is 3 . 
The obtained system of equation of motion is a mixed system of differentialalgebraic equation. We can solve it by using numerical methods. The discussion of solving of the system of differential algebraic equations is out of scope of this article, but in general we can use some of implicit Runge-Kutta methods or Gear's algorithms for stiff system, see e.g. [5], [8], [13], [14] etc. For this purpose the software DAESOL is developed at the Department of Applied Mechanics of the Hanoi University of Technology. The package is easy-to-use with possibility to have the interface with other worldwide softwares.

\section{Conclusion}

In the article we have discussed and developed one new computer method for constructing the equation of motion of constrained mechanical systems. The constraints are appended successively to the system, therefore the equation of motion of each stage is constructed. Using this algorithm we can investigate the constraints and the mechanical system more in detail. The technique is convenient for computer generation but for simple cases the equation can be written by hand too.

Note that it is possible to combine our technique with other methods for constructing equation of motion. It means that in the step 1 of both above algorithms any methods could be used and the obtained form of equations of motion can serve as the free system.

This publication is completed with financial support from the National Basic Research Program in Natural Sciences.

\section{REFERENCES}

1. de Jalon G. J., Bayo E. Kinematic and dynamic simulation of multibody systems: The real-time challenge. Springer, NewYork/Berlin 1996.

2. Dinh Van Phong. Principle of compatibility and criteria of ideality in study of constrained mechanical systems. Strojnicky casopis (Journal of Czech and Slovak mechanical engineering), 47, 1996, N.1, pp. 2-11, Bratislava.

3. Dinh Van Phong. An algorithm for calculating reaction forces in constrained mechanical systems. Inzenyrska mechanika (Engineering Mechanics), 5, 1998, N.4, Prague, pp. 291-297.

4. Do Sanh. On the motion of constrained mechanical systems. The thesis of doctor of science, Hanoi University of Technology, Hanoi, 1984.

5. Do Sanh, Dinh Van Phong. The principle of compatibility and computational mechanics. Proceedings of the NCST of Vietnam, Vol. 7, N. 1, pp. 3-8, 1995.

6. Kane T. R., Levison D. A. Dynamics: theory and application. McGraw Hill, 1985. 
7. Haug E. J. Intermediate Dynamics. Prentice Hall, Englewood Cliffs, 1992.

8. Nguyen Nhat Le, Dinh Van Phong, Do Sanh. On numerical methods for constrained mechanical systems. Proceedings of the IUTAM Symposium on recent development in non-linear oscillation of mechanical system. Kluwer Academic Publisher, Dodrecht/Boston/London, 1999. pp. 207-216.

9. Nikravesh P. E. Computer-Aided analysis of mechanical system. Prentice-Hall, Englewood Cliffs, 1988.

10. Schiehlen W. Multibody system dynamics: Roots and perspectives. Multibody system dynamics 1: 149-188, Kluwer Academic Publishers, 1997.

11. Shabana A. A. Computational Dynamics. John Willey \& sons, NewYork, 2001.

12. Soellner E. E., Fuhrer C. Numerical methods in multibody dynamics. Teubner Stuttgart, 1998.

13. Stejskal V., Valasek M. Kinematics and dynamics of machinery. Marcel Dekker, New York, 1996.

Received November 5, 2001

THUẬT GIẢI HỒI QUY CHO VIỆC XÂY DỰG PHƯƠNG TRİNH CHUYỂN ĐỘNG CỦA CÁC HỆ CƠ HỌC CHỊU LIÊN KẾT

Bài báo trình bày phương pháp xây dựng phương trình chuyển đụ̣̂̂ng cho các cơ hệ chịu liên kết. Các liên kết được xét đến một cách lần lượt và được đưa thêm vào hệ đã được xây dựng trước đó. Với cách này thuật giải sẽ rất mềm dẻo và cho phép khảo sát từng liên kết một cách chi tiết. Một ví dụ đơn giản được phân tích kỹ lưỡng để minh họa cho từng bước của thuật giå̉i.

Address:

Dinh Van Phong

Department of Applied Mechanics, Hanoi University of Technology

1st Dai Co Viet, Hanoi, Vietnam

Tel: 8680469 Home: 8536298

Email: phong@mail.hut.edu.vn 